\title{
Synthesis of Carboxyl-EDOT as a Versatile Addition and Additive to PEDOT:PSS
}

Daniele Mantione, ${ }^{\dagger}$ Ariana Villarroel Marquez, ${ }^{\dagger}$ Federico Cruciani, $^{\dagger}$ Cyril Brochon, $^{\dagger}$ Eric Cloutet $^{+*}$ and Georges Hadziioannou ${ }^{+*}$

†Laboratoire de Chimie des Polymères Organiques (LCPO - UMR 5629), Bordeaux INP, Université de Bordeaux, CNRS, 16 Av. Pey-Berland, 33607, Pessac, France.

*Corresponding Authors: Prof. Eric Cloutet cloutet@enscbp.fr, Prof. Georges Hadziioannou hadzii@enscbp.fr

KEYWORDS: carboxyl-EDOT, PEDOT, cross-link, conducting polymers, electronic material

ABSTRACT: Two steps synthesis of EDOT (3,4-ethylenedioxythiophene) derivate bearing a carboxylic acid group (carboxyl-EDOT) is presented. This reactive monomer has been copolymerized with EDOT to afford PEDOT copolymers. Thanks to the most common additives usually added to the PEDOT:PSS dispersion, ethylene glycol and 4-Dodecylbenzenesulfonic acid (DBSA), the carboxylic acid has been used to crosslink the material via esterification reactions. This result offers the possibility to produce a polymer network without adding any crosslinking agent. Furthermore, the short synthetic pathway of carboxyl-EDOT offers the possibility to 
incorporate new functionality either in EDOT monomer or in PEDOT materials with a reasonable chemical effort and background.

Conductive polymers, bioelectronic, organic electrochemical transistor (OECT) etc. these and more terms rapidly entered ubiquitarians in the scientific community in the last decades. ${ }^{1}$ The golden standard material, omnipresent in a large variety of devices is poly 3,4ethylenedioxythiophene (PEDOT). ${ }^{2}$ Despite the large use of this material, thanks to its mechanical and electrochemical properties, ${ }^{3}$ the variation of the bare chemical structure of the monomer EDOT is still an open challenge. Due to the difficulties in the synthetic part and the ease to obtain and use the commercial PEDOT, often, this conductive polymer, and more precisely, the commercial aqueous dispersion of PEDOT:PSS (PEDOT: PolyStyrene Sulfonate) is considered as an unmodifiable part. Another key point that turned PEDOT:PSS dispersion in the most used in the electronic field, is the widely biocompatibility in vivo and in vitro. ${ }^{4-6}$ Related to these bioapplications, crosslinking is needed to avoid re-dispersion/dissolution of the conducting film.,8 In order to overcome this problem, various processing methods were used, such as: UV light or the use of polyethylene oxide, DVS (divinylsulfone) or GOPS (3glycidoxypropyltrimethoxysilane).${ }^{9-12}$ All these methods have certain pros and cons; among these, GOPS is the most used one, even if it requires high curing temperature $\left(140{ }^{\circ} \mathrm{C}\right.$ for $\left.1 \mathrm{~h}\right)$ and has negative effect on the conductivity values, due to the natural insulated properties of the siloxane network. To overcome this outcome, a large variety of additives are added; among them, ethylene glycol and DBSA are the most used. Ethylene glycol is known to increase charge density and mobility, ${ }^{13,14}$ and DBSA gives wetting and bulky-dopant properties. ${ }^{13,15,16}$ As we can easily see, we have in the same mixture a difunctional molecule, the diol, and a strong organic acid, the 
benzenesulfonic. These two reagents plus a carboxylic acid can led to a difunctional ester, as omnipresently reported in organic chemistry books. EDOT derivate bearing carboxylic acid are poorly present in literature counting only two examples. ${ }^{17-20}$ The first carboxyl-EDOT has been reported by Prof. Martin’s group ${ }^{17,18}$ and in the second case Prof. Mecerreyes' group showed a close derivate named ProDOT-COOH. ${ }^{19,20}$

ProDOT derivatives are easier to synthesize but conductivity and mechanical properties are known to be lower than EDOT. In Martin's case, the low yield and the high number of organic chemistry steps leads to poor yields and not easy handling.

In this work we present a new two steps synthetic route to get carboxyl-EDOT, with an overall yield of about $70 \%$ as shown in Scheme 1.

a)<smiles>COC(=O)C(O)CO</smiles>

1<smiles>COc1cscc1OC</smiles>
$\underset{100^{\circ} \mathrm{C}, 2 \text { days }}{\stackrel{\text { Toluene, } \mathrm{Ar}}{\longrightarrow}}$<smiles>COC(=O)C1COc2cscc2O1</smiles>

2

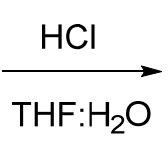

THF: $\mathrm{H}_{2} \mathrm{O}$

Scheme 1. Synthetic route to carboxyl-EDOT (3). The reaction consists in two steps: a)

Methyl glycerate (1) reacting with 3,4-dimethoxythiophene; b) Intermediate (2) hydrolysis using $\mathrm{HCl}$ in a mixture THF-water.

With the carboxylic derivate of EDOT available, we explore the possibility to use the carboxylic moiety to avoid the addition of any crosslinking agent. We copolymerized EDOT together with carboxyl-EDOT in three different concentrations: 5, 10 and 15 mol\% (Chapter 5 SI) using PSS as anionic counterpart chain. The reaction has been performed via oxidative chemical polymerization using ammonium persulfate as oxidant and Iron (III) as catalyst, in water for 8 hours at room temperature. In order to mimic as much as possible the commercial PEDOT:PSS 
(Clevios $\left.\mathrm{P}{ }^{\circledR}\right),{ }^{21}$ and to prove if the presence of the carboxylic moiety is disturbing the well-known behavior of the conductive dispersion, $1.3 \%$ s.c.(solid content), with a ratio between PEDOT and PSS of 1 to 2.5 have been chosen. UV-Vis-NIR analysis has been used to first check the resulting dark blue dispersions. The spectra (Figure S1) show only two strong characteristic bands of PEDOT:PSS, around $900 \mathrm{~nm}$ and above $1200 \mathrm{~nm}$, derived from the radical cations and dications respectively. This sustains that the presence of the carboxylic group is not affecting the doped state of PEDOT, as the band of the neutral chain (the undoped or rather not conductive) below $600 \mathrm{~nm}$, is totally absent, as in the bare PEDOT:PSS. ${ }^{22}$ Varying the concentration of carboxyl-PEDOT the spectra still look very similar: further confirmation that the carboxylic is not disrupting the doping effect. To truly confirm the incorporation of the carbonyl compound and due to the difficulties to analyze PEDOT dispersion, dialysis has been done on the samples after polymerization, checking the absence of any EDOT derivates dialysate. ${ }^{18,23}$ Having the copolymerized dispersion, we took advantage of two widely used additives: ethylene glycol and DBSA. The diol provides a difunctional block and DBSA an acid catalysis, in order to perform an in-situ esterification between PEDOT chains, as resumed in Figure 1. The mixtures are prepared by mixing PEDOTs dispersions, with the desired amount of ethylene glycol and a catalytic amount of DBSA, in our case we used $20 \% \mathrm{v}$-v of ethylene glycol. The chosen quantity of ethylene glycol has been chosen in order to get the best physicochemical properties and introduced in excess, ${ }^{24,25}$ anyhow low concentration of Ethylene Glycol (EG) with respect to EDOT-Acid could be used giving rise to similar crosslinking behavior (see Table S2). In the case of DBSA, the residual acidity of PSS does not allow the reaction using these conditions and to get an insoluble film its addition was mandatory. The resulting blends were sonicated and used right after (for a better reproducibility, we have avoided to store the prepared mixtures) to make drop-casted or spin coated films. The 
resulting films are insoluble in water: to prove that, we left two drop-casted films, one with DBSA and one without, drying overnight and we tested the solubility in Phosphate-buffered saline (PBS) (Figure S3). Among the three different suspensions we tested (5, 10, 15\% of carboxyl-EDOT in respect to EDOT), below 10\% of carboxyl-EDOT the crosslinking is ineffective, although 10 and $15 \%$ resulted very similar.

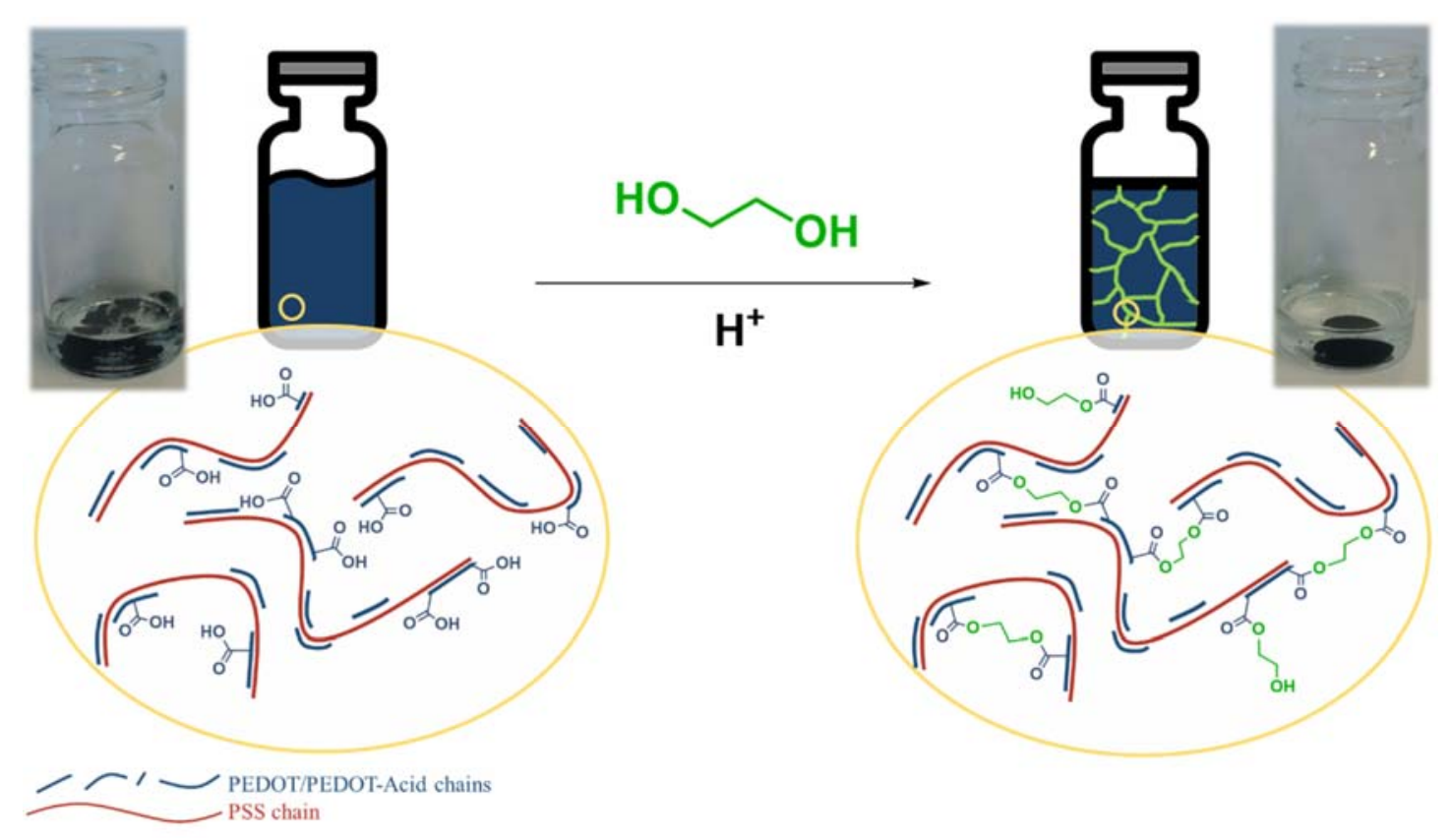

Figure 1. Schematic representation of PEDOT/carboxyl-PEDOT:PSS dispersions, before (left) and after (right) crosslinking by ethylene glycol in acid environment.

The detailed reaction is shown in Scheme 2: ethylene glycol is bridging two PEDOT chains.

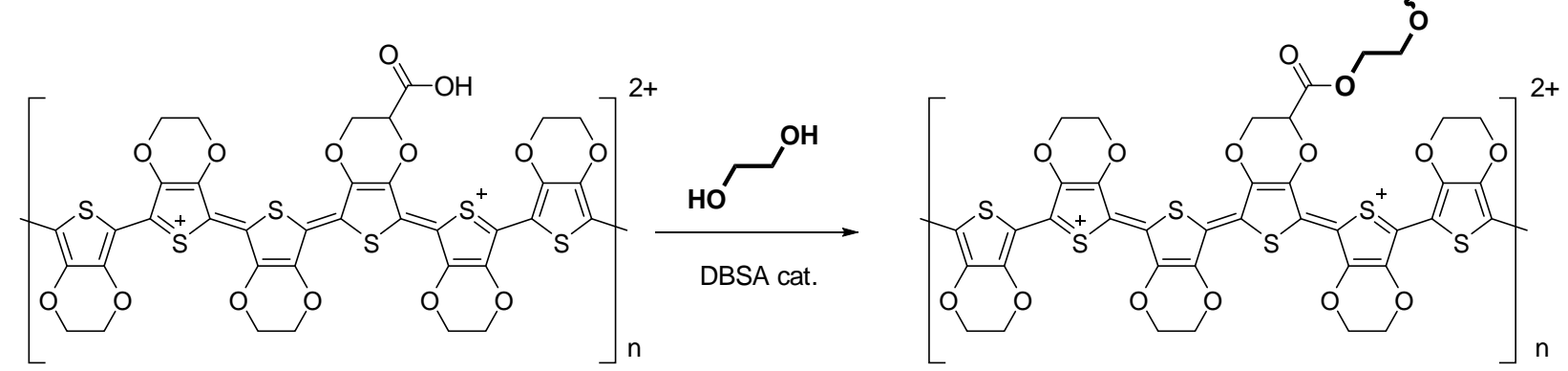

Scheme 2. Esterification reaction scheme of PEDOT/carboxyl-PEDOT (PSS is omitted for clearness). 
Attenuated Total Reflectance-Fourier Transform InfraRed spectroscopy (ATR-FTIR) (Figure S2) has been used to characterize the resulting insoluble films. To check the presence of the carboxylic moiety and the possible transesterification reaction, analyses of the dispersion and the films, before and after crosslinking have been realized. Varying the quantity of carboxyl-EDOT does not affect the infrared, showing almost identical profiles for all the three compositions. The expected band at $1700 \mathrm{~cm}^{-1}$ is not present, covered by the noise of PEDOT:PSS; anyhow, an enhanced band at around $1250 \mathrm{~cm}^{-1}$ being related to the $\mathrm{C}-\mathrm{O}$ stretching of the carboxylic acid could be detected. Moreover, in the crosslinked film, this band move to higher frequency, with a more definite shape, characteristic of ester molecules. Conductivity has been measured and it is confirming that carboxyl-PEDOT is not affecting the electrochemical behavior giving values almost identical to PEDOT:PSS treated with ethylene glycol, of about 200 S/cm, reported in literature (Table S2). ${ }^{24-}$ 26

In this paper we have designed a new two-steps synthesis to afford an outstanding EDOT derivate, called carboxyl-EDOT and bearing a reactive carboxylic acid on the oxolane ring. We have used this molecule to copolymerize with EDOT, creating different dispersion of PEDOT/carboxylPEDOT:PSS. With the aim to avoid the adding of any crosslinking agent. We exploited the carboxylic function together with two general additives broadly used in literature: ethylene glycol and DBSA. In this way we arrived to a crosslinked, insoluble material, thanks to the acid catalyzed esterification occurring between the diol and two different molecules present in the chain of carboxyl-PEDOT. To ensure the maintain of the PEDOT:PSS physical-chemical properties, we characterized the carboxyl-PEDOT dispersions via UV-Vis-NIR and the films via ATR-FTIR, 
confirming that carboxyl-EDOT is not affecting the overall properties of the dispersion. These findings can be applied in bioelectronics devices to avoid the use of crosslinking agents, making the carboxyl-EDOT derivate prone to any further functionalization either in the monomer or in film state.

\section{ASSOCIATED CONTENT}

\section{Supporting Information.}

Materials and methods, ATR-FTIR spectra, UV-Vis-NIR spectra, picture of not cross-linked and cross-linked films behavior in PBS solution, synthesis and NMR spectra of 2, 3, of PEDOT:PSS and PEDOT/carboxyl-PEDOT:PSS, polymerization conditions, film formation and electrical values are available free of charge via the internet at http://pubs.acs.org.

\section{AUTHOR INFORMATION}

\section{Corresponding Authors}

*E-mail: Prof. Eric Cloutet cloutet@enscbp.fr, Prof. Geroges Hadziioannou hadzii@enscbp.fr.

\section{ACKNOWLEDGMENT}

The authors thanks Iñaki Gomez (Cidetec, Spain) and Elena Righini (IULM, Italy) for fruitful talks and availability. The LCPO-support: Aude Manson, Melanie Bousquet, Ellena Karnezis and Gilles Pecastaings for endless everyday help. We are also thankful to ELORPrintTec ANR-10-EQPX28-01 and to LCPO/Arkema/ANR INDUSTRIAL CHAIR "HOMERIC” ANR-13-CHIN-000201.

\section{References}


(1) Rivnay, J.; Owens, R. M.; Malliaras, G. G. Chem. Mater. 2014, 26 (1), 679-685.

(2) Casado, N.; Hernández, G.; Sardon, H.; Mecerreyes, D. Prog. Polym. Sci. 2016, 52, $107-$ 135.

(3) Elschner, A.; Kirchmeyer, S.; Lövenich, W.; Merker, U.; Reuter, K. PEDOT, 1st ed.; CRC Press: Boca Raton FL USA, 2010.

(4) Mantione, D.; del Agua, I.; Sanchez-Sanchez, A.; Mecerreyes, D. Polymers (Basel). 2017, 9 (12), 354.

(5) Khodagholy, D.; Doublet, T.; Quilichini, P.; Gurfinkel, M.; Leleux, P.; Ghestem, A.; Ismailova, E.; Hervé, T.; Sanaur, S.; Bernard, C.; Malliaras, G. G. Nat. Commun. 2013, 4, 1575.

(6) Mawad, D.; Artzy-Schnirman, A.; Tonkin, J.; Ramos, J.; Inal, S.; Mahat, M. M.; Darwish, N.; Zwi-Dantsis, L.; Malliaras, G. G.; Gooding, J. J.; Lauto, A.; Stevens, M. M. Chem. Mater. 2016, 28 (17), 6080-6088.

(7) Nezakati, T.; Seifalian, A.; Tan, A.; Seifalian, A. M. Chem. Rev. 2018, 118 (14), 67666843

(8) Colucci, R.; Quadros, M. H.; Feres, F. H.; Maia, F. B.; de Vicente, F. S.; Faria, G. C.; Santos, L. F.; Gozzi, G. Synth. Met. 2018, 241, 47-53.

(9) Xing, Y.; Qian, M.; Wang, G.; Zhang, G.; Guo, D.; Wu, J. Sci. China Technol. Sci. 2014, 57 (1), 44-48.

(10) Huang, T.-M.; Batra, S.; Hu, J.; Miyoshi, T.; Cakmak, M. Polymer (Guildf). 2013, 54 (23), 6455-6462. 
(11) Mantione, D.; del Agua, I.; Schaafsma, W.; ElMahmoudy, M.; Uguz, I.; Sanchez-Sanchez, A.; Sardon, H.; Castro, B.; Malliaras, G. G.; Mecerreyes, D. ACS Appl. Mater. Interfaces 2017, 9 (21), 18254-18262.

(12) Zhang, S.; Hubis, E.; Girard, C.; Kumar, P.; DeFranco, J.; Cicoira, F. J. Mater. Chem. C 2016, 4 (7), 1-4.

(13) Ouyang, J.; Xu, Q.; Chu, C.-W.; Yang, Y.; Li, G.; Shinar, J. Polymer (Guildf). 2004, 45 (25), 8443-8450.

(14) Jhe-Yow-Jon, L.; Wei-Shih, N.; Jhe-You, L. J. Appl. Phys. 2015, 117 (21), 215501.

(15) Crispin, X.; Marciniak, S.; Osikowicz, W.; Zotti, G.; van der Gon, A. W. D.; Louwet, F.; Fahlman, M.; Groenendaal, L.; De Schryver, F.; Salaneck, W. R. J. Polym. Sci. Part B Polym. Phys. 2003, 41 (21), 2561-2583.

(16) Mukherjee, A. K.; Thakur, A. K.; Takashima, W.; Kaneto, K. J. Phys. D. Appl. Phys. 2007, 40 (6), 1789-1793.

(17) Povlich, L. K.; Cho, J. C.; Leach, M. K.; Corey, J. M.; Kim, J.; Martin, D. C. Biochim. Biophys. Acta - Gen. Subj. 2013, 1830 (9), 4288-4293.

(18) Wei, B.; Liu, J.; Ouyang, L.; Kuo, C.-C.; Martin, D. C. ACS Appl. Mater. Interfaces 2015, 7 (28), 15388-15394.

(19) Mantione, D.; Casado, N.; Sanchez-Sanchez, A.; Sardon, H.; Mecerreyes, D. J. Polym. Sci. Part A Polym. Chem. 2017, 55 (17), 2721-2724.

(20) Marina, S.; Mantione, D.; ManojKumar, K.; Kari, V.; Gutierrez, J.; Tercjak, A.; SanchezSanchez, A.; Mecerreyes, D. Polym. Chem. 2018, 9 (27), 3780-3790. 
(21) Xia, Y.; Ouyang, J. ACS Appl. Mater. Interfaces 2012, 4 (8), 4131-4140.

(22) Massonnet, N.; Carella, A.; Jaudouin, O.; Rannou, P.; Laval, G.; Celle, C.; Simonato, J.-P. J. Mater. Chem. C 2014, 2 (7), 1278.

(23) Jennings, M.; Kendrick, I.; Green, C.; Lustig, S. R. EMBARK 2018, $2,4$.

(24) Zhang, S.; Kumar, P.; Nouas, A. S.; Fontaine, L.; Tang, H.; Cicoira, F. APL Mater. 2015, 3 (1), 014911.

(25) Crispin, X.; Jakobsson, F. L. E.; Crispin, a; Grim, P. C. M.; Andersson, P.; Volodin, a; van Haesendonck, C.; Van der Auweraer, M.; Salaneck, W. R.; Berggren, M. Chem. Mater. 2006, 18 (4), 4354-4360.

(26) ElMahmoudy, M.; Inal, S.; Charrier, A.; Uguz, I.; Malliaras, G. G.; Sanaur, S. Macromol. Mater. Eng. 2017, 302 (5), 1600497. 
TOC:
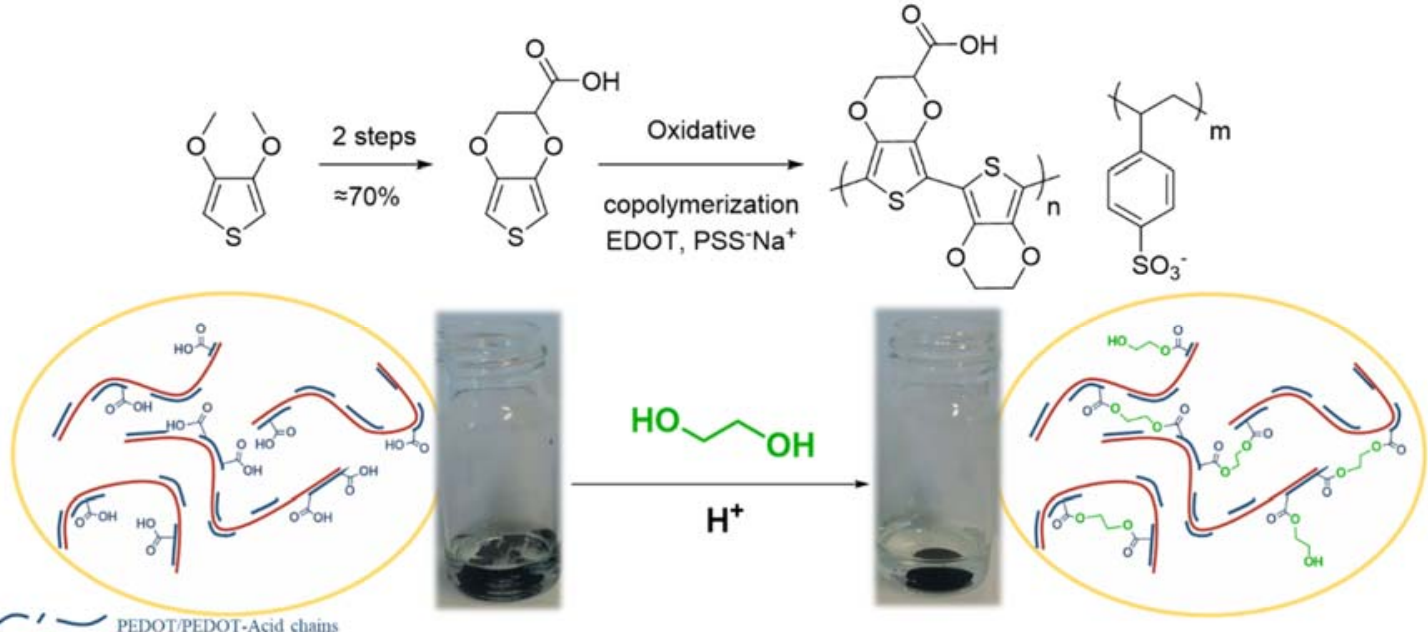